\title{
Observation of Aerosol Generation by Human Subjects During Cardiopulmonary Exercise Testing Using a High-powered Laser Technique
}

Christopher M. Varga

Vyaire Medical

Keith J. Kwiatkowski

Vyaire Medical

Michael J. Pedro

Vyaire Medical

Herman Groepenhoff

Vyaire Medical

Edward A Rose ( $\nabla$ erose@20poms.com )

Vyaire medical https://orcid.org/0000-0001-7086-8639

Callum Gray

LaVision $\mathrm{GmbH}$

Kai D. Pinkerton

Vyaire Medical

Michael G. McBride

CHOP: The Children's Hospital of Philadelphia

Stephen M. Paridon

CHOP: The Children's Hospital of Philadelphia

\section{Research Article}

Keywords: Aerosols, cardiopulmonary exercise testing, physiological measurement

Posted Date: July 1st, 2021

DOI: https://doi.org/10.21203/rs.3.rs-650367/v1

License: (1) This work is licensed under a Creative Commons Attribution 4.0 International License. Read Full License 


\section{Abstract}

Purpose: Human respiratory aerosols may have important implications for transmission of pathogens. The study of aerosol production during vigorous breathing activities such as exercise is limited. Data on aerosol production during cardiopulmonary exercise testing (CPET) are lacking.

Methods: We used a high-powered, pulsed Nd:YAG laser to illuminate a region of interest in front of two healthy adult subjects during CPET. Subjects exercised to the point of respiratory compensation. Images were captured with a high-speed, high-resolution camera to determine net exhaled particle (NEP) counts at different phases of $\mathrm{CPET}$, including resting breathing, submaximal exercise, peak exercise, and active recovery. Experiments were performed with the room ventilation activated.

Results: NEP counts remained relatively constant until late/peak exercise when they decreased prior to rebounding into recovery. NEP counts at resting breathing were higher than those reported using other methods of measurement. Exhaled particles were in the submicron size range.

Conclusion: Our method of aerosol particle quantification enables measurement of significant quantities of ultrafine particles and dynamic assessment of aerosol production during CPET. The unique pattern of aerosol production observed during submaximal and peak exercise suggests that extension of results from resting breathing to CPET may not be appropriate.

\section{Introduction}

Human breathing produces aerosols which populate the surrounding ambient environment.[1] Depending on the nature of the expelled breaths (such as resting, vigorous, or coughing), the size of the exhaled particles, and ambient environmental factors (temperature, humidity, air currents), the persistence of these aerosols in the environment may be either short or prolonged.[2,3] Exposure to such aerosols for persons in the immediate surrounding environment can potentially be harmful if the aerosol carries infectious material.[4] Widespread concern over transmissibility of viruses via respiratory droplets has created a tremendous and renewed focus on aerosol generation during various activities and medical procedures. Numerous studies measuring humangenerated aerosols have been carried out [4-12] and in general these studies have primarily focused on aerosols generated during common person-to-person interactions at rest such as talking, coughing, and sneezing. Published studies of aerosols generated during vigorous breathing activities (exercise) on the other hand are limited.

Cardiopulmonary exercise testing (CPET) provides assessment of pulmonary and cardiovascular system functionality by measuring the response of these systems to both submaximal and peak effort during exercise. [13] Concerns regarding exposure of healthcare workers and families to aerosols produced during spirometry and numerous other medical procedures have led to strict regulations on CPET testing labs by expert panels.[7, 13-20] However, measurement and analysis of aerosols produced during CPET are lacking, and applicability of aerosol data from resting breathing and spirometry maneuvers to CPET have not been adequately established.

In this study we measured aerosol generation by healthy human subjects using a novel high-powered pulsedlaser technique with the primary objective of comparing aerosol concentrations generated during CPET at 
submaximal and peak exercise to those generated during resting tidal breathing. To our knowledge, this is the first study to objectively measure aerosol generation during CPET.

\section{Methods}

Two healthy, human CPET trainers participated as test subjects in the present study after providing informed consent. The testing protocol is consistent with the principles of the Declaration of Helsinki[21] and was approved by Vyaire Medical Affairs. Experiments were carried out in a diagnostic training lab at Vyaire Medical in Mettawa, IL, a leased space in a commercial office building. The building air conditioning (A/C) system complies with the International Mechanical Code, ASHRAE Standard 90.1, and the International Energy Conservation Code as mandated by the State of Illinois.

The experimental setup is shown in Figure 1 (top). A gantry was built to hold a flashlamp-pumped, Q-switched double-cavity pulsed Nd:YAG laser (Quantel, Bozeman, MT) above the subject. A 90-degree beam-steering mirror and a variable-beam-waist optic (LaVision USA, Ypsilanti, MI) were utilized to direct and convert the laser beam into a light sheet to illuminate aerosol particles in the region of interest (ROI). A 5.5-megapixel CMOS camera (LaVision USA, Ypsilanti, MI) with global shutter and double-frame mode was combined with a 60mm f2.8 macro lens (Nikon USA, Melville, NY). Image acquisition was synchronized with the laser pulses using a PTU-X Synchronizer (LaVision USA, Ypsilanti, MI). The imaging ROI was located immediately downstream of the CPET interface to capture particles generated on a breath-by-breath basis.

Particle imaging data were captured and processed using commercial image-processing software (DaVis 10, LaVision USA, Ypsilanti, MI). Image pairs were captured at $15 \mathrm{~Hz}$ for durations selected to match the test being performed. Particle-image velocimetry (PIV) was used to measure particle velocities. Velocities of the flows downstream of the CPET patient-interface were utilized to assign particle images to either inhalation or exhalation. Particle counts were calculated for each image using a multi-step smoothing and pixel-threshold algorithm. Figure 1 (bottom) shows an example raw particle image prior to processing (left) and a processed image (right).

\section{CPET Breath-by-Breath Measurements}

CPET with breath-by-breath aerosol particle counting was performed using two different cycle-based CPET systems and associated patient interfaces. The first system consisted of a Vyntus ${ }^{\mathrm{TM}}$ CPX Metabolic Cart (Vyaire Medical, Mettawa, IL) using a Digital Volume Transducer (DVT) flow sensor (Vyaire Medical, Mettawa, IL) with head attachment of a 7450 Silicone Oro-Nasal mask (Hans Rudolph Inc, Shawnee, KS). The second system consisted of a Vmax ${ }^{\mathrm{TM}}$ Metabolic Cart (Vyaire Medical, Mettawa, IL) using a hot-wire mass flow sensor (MFS, Vyaire Medical, Mettawa, IL), and mouthpiece and nose clip (VacuMed, Ventura, CA) secured to the subject with headgear (Vyaire Medical, Mettawa, IL). An upright cycle ergometer (Ergoline GmbH, Bitz, Germany) and Cardiosoft 12-lead ECG (GE Healthcare, Chicago, IL) were common to both systems. The DVT and MFS were secured in place such that the laser sheet could be aligned precisely with the outlet of each sensor. The subjects breathed through an extension tube placed between the flow sensor and the mask or mouthpiece.

Figure 2 provides a schematic and a camera image of the experimental setup and particle counting ROI for the CPET experiments. 
Two healthy trained subjects exercised using a 3-phase incremental ramp protocol (2 minutes resting breathing, 30-watt ramp exercise, 2-minute active recovery) until volitional stoppage due to exhaustion when the subject reached respiratory compensation. Particle images were taken in sequences of 200 image pairs at 90 seconds resting breathing, at 2-minute intervals during exercise, and at reaching peak work-rate intensity. Following this, images were captured at 30 seconds and 2 minutes during recovery.

Estimation of endogenous aerosol particle generation during the testing followed a process of assigning particle counts to either inhalation or exhalation using measured velocities, followed by integration over each breath to determine the aerosol particles per unit volume generated at each point in the CPET regimen. Figure 3 contains a representative raw particle count (blue) and mean velocity plot (orange) from the breath-by-breath CPET measurements at peak exercise. Particle count and mean velocity are plotted as a function of time. Inhalation and exhalation phases can be clearly observed as the particle count rises to a maximum during exhalation and returns to a baseline value during inhalation. The transition from inhalation to exhalation occurs as the mean velocity changes from positive (inhalation) to negative (exhalation).

Measured particle counts were integrated over each breath (area under the curve, AUC) to calculate the aerosol concentration in the interrogation region for both inhalation and exhalation. Each exhalation of particles includes both endogenously generated aerosol particles plus particles inhaled from the surrounding ambient region on the prior inhalation. Therefore, the net amount of aerosol generated per breath was determined by subtracting the particle count of the prior inhalation from each measured exhalation count. The thickness of the laser sheet was measured optically such that the illuminated volume could be calculated as the area of the rectangular ROI shown in Figure 2 multiplied by the laser sheet thickness. This volume was $2.4 \mathrm{~cm}^{3}$ and enabled scaling of all aerosol particle counts to a per $\mathrm{ml}$ basis.

Aerosol Size

A qualitative comparison of aerosol particles generated by the test subjects during CPET with both side-stream tobacco cigarette smoke particles and jet nebulizer droplets enabled estimation of aerosol size; Figure 4 compares images of these aerosols. Aerosol droplets generated with a jet nebulizer (MistyFast, Vyaire Medical, Mettawa, IL) have a known size range of 2-3 microns[22] and are observably much larger than the CPET aerosol particles. On the other hand, the tobacco smoke particles (with known size range of 0.1-0.3 microns[23]) appear very similar in size to the CPET-generated particles. Based on this similarity, we estimate that the aerosols generated in this study during CPET are primarily in the lower sub-micron range $(<0.3$ microns) which is consistent with other published literature.[24-28]

\section{Results}

Demographic data for the two experimental subjects (designated PP1 and PP2) is provided in Table 1. Timing between subject tests was irregular and varied from 10 minutes to 2 hours. Table 2 contains subject exercise test data from the breath-by-breath CPET experiments at resting breathing and peak exercise. Figure 5 contains plots of net exhaled particles (NEP) per $\mathrm{ml}$ as a function of exercise phase during CPET for both subjects and interfaces (DVT - top, MFS - bottom). Minute ventilation for each subject is overlayed in each plot. For both CPET interfaces/flow sensors and both subjects, the NEP per $\mathrm{ml}$ reached a minimum value at peak exercise and subsequently increased into recovery. NEP per $\mathrm{ml}$ was two to three times higher at resting breathing than 
at peak exercise. On a per minute basis (Figure 6), NEP production initially rose with exercise but declined in late and peak exercise, then rebounded in early recovery to values approximately three to four times higher than measured during resting breathing.

Figure 7 contains a comparison plot of NEP per $\mathrm{ml}$ as a function of CPET phase for subject PP1 with the two different CPET interfaces/flow sensors. There was a small but measurable difference in NEP between the two CPET interfaces; the trend of NEP per $\mathrm{ml}$ as a function of CPET phase (i.e., reaching a minimum at peak exercise and then rising during recovery) was observed to be independent of CPET interface/flow sensor.

\section{Discussion}

In this experimental study, the first of its kind to objectively measure endogenous aerosol production during CPET, we report two important physiological findings. Firstly, we observed a unique pattern of endogenous aerosol production as subjects transitioned from resting breathing through submaximal exercise to peak exercise, followed by recovery. Exhaled aerosol particle concentration remained relatively constant until late/peak exercise, when it decreased. During recovery the concentration then rebounded to approximately resting breathing levels. This pattern runs counter to the expectation that increased effort should lead to increased aerosol production. The reasons for this pattern are unclear but may involve relative dehydration of the airways or earlier clearance of secretions during the submaximal portion of the testing protocol. This pattern of aerosol production has not been previously noted with other types of resting respiratory maneuvers. [24] The reasons for the rebound in exhaled aerosol particle concentration in recovery are also unclear but may be related to changes in tidal volume and physiologic dead space ventilation. These data suggest that extrapolation of aerosol production results from resting breathing maneuvers $[2,6,27,29-31]$ to CPET, particularly at peak effort levels, may not be appropriate.

Secondly, we observed substantially higher exhaled particle concentrations (1-2 orders of magnitude) during both resting breathing and exercise in comparison to published literature, a result which we believe is attributed primarily to measurement technique and test setup. Studies in the literature have shown discrepancies of several orders of magnitude in reported concentrations of aerosols in exhaled breath.[25, 27, 29, 32, 33] The high-powered laser technique used in our study coupled with the relatively small area of focused illumination enabled counting of significant numbers of particles less than 0.3 microns in size. This is evidenced by a direct comparison of illuminated exhaled aerosol during CPET to sidestream tobacco smoke particles measured under the same setup (Figure 4). Sidestream tobacco particles are well-established to be in the $0.1-0.3$ micron size range.[23] This in effect allows us to 'see' aerosol particles that may remain invisible to techniques used in previously published aerosol counting studies. For example, we note that in the experiments of Asadi et al[32], the authors reported significantly lower exhaled aerosol concentrations during human talking than those measured in the present study during various phases of CPET. However, the instrument used in those experiments (light-scattering spectrometer, TSI Model 3321) has a stated minimum aerodynamic particle sizing limit of 0.5 microns. For this reason, direct quantitative comparison of measured aerosol concentrations is not appropriate in this case nor in numerous other published studies due to differences in both technique and instrument. 
Furthermore, we note that exhaled aerosol particles are in the same size range as persistent ambient background aerosols which underscores the importance of appropriately accounting for inhaled particle counts. Our novel use of particle velocity measurements to assign aerosol counts to inhalation and exhalation phases enabled us to improve estimation of true exhaled aerosol concentration. Although there is known heterogeneity between subjects in exhaled aerosol concentration measurement[12], this in itself is not sufficient to explain order of magnitude differences which we propose are fundamentally attributed to instrument and technique. The existence of larger quantities of ultrafine particles in exhaled breath during both resting breathing and various levels of exercise is physiologically important, and likely has clinically relevant implications in a number of important areas. We do not venture at this time to speculate on these; ideally our current findings will encourage additional experiments and related physiological studies.

It is also worth noting that there is essentially an absence of larger particle production during tidal breathing with CPET, as demonstrated in Figure 4. This is in contrast to previous studies with resting respiratory maneuvers such as phonation, forced exhalation, or coughing. The reasons for this difference are unclear but may be related to the relatively low exhalation pressures seen in the tidal volume breathing with CPET even at peak exercise (Figure 3) compared to these more forceful resting exhalations such as coughing. Dehydration, as stated above, could also be a factor. These differences will need further study to assess both their etiology and potential significance.

Our study has certain limitations. There are no similar studies in the available literature for direct external validation due to our unique method of quantifying aerosol particle production during CPET. Previous studies have analyzed subjects using different technologies and measurement setups, and our data suggest that it would be imprudent to extrapolate those studies to CPET. We did not directly measure the size of particles being produced but leveraged surrogates of particle size, namely smoke[23] and nebulized water[22]. Most importantly, we had only a small number of test subjects, limiting generalizability of findings and increasing the chances of bias. We believe our findings warrant further experimental studies of aerosol production during CPET.

\section{Conclusion}

The results of the present study describe a physiologically interesting pattern of aerosol production during CPET wherein net exhaled particle counts decrease around peak exercise to levels significantly below those at resting breathing. This suggests that extension of resting breathing aerosol data to CPET may not be appropriate. Exhaled particle concentrations were substantially higher in our experiments than those previously reported, a result which may be attributed to the capability of our measurement technique to capture ultrafine submicron aerosol particles.

\section{Declarations}

\section{Acknowledgments}

CMV takes final responsibility for, and is the guarantor of, the content of this manuscript, including the data and analysis. 
The authors wish to express their appreciation to Edward A. Rose for his assistance with the manuscript.

Funding: The laboratory space, equipment, and administrative support were provided by Vyaire Medical. Vyaire Medical had no role in the design of the study nor the interpretation of its results.

Conflicts of interest/Competing interests. CMV, KJK, MJP, HG, and KDP are employees of Vyaire Medical, Mettawa, IL.; CG is an employee of LaVision Inc., Ypsilanti, MI.

Availability of data and material: All data is available upon request to corresponding author.

Code availability. Not applicable

Authors' contributions: All authors made substantial contributions to the design, acquisition, analysis, and interpretation of the work; were involved in drafting and revising the manuscript; and grant final approval of this version. All authors agree to be accountable for all aspects of this report in ensuring that questions related to the accuracy or integrity of any part of this work were appropriately investigated and resolved.

Ethics approval: The testing protocol is consistent with the principles of the Declaration of Helsinki and was approved by Vyaire Medical Affairs.

Consent to participate: Two authors participated as test subjects in the present study after providing informed consent.

Consent for publication: All authors have given consent to the publication of this work.

\section{References}

1. Duguid, J. P. (1945). The numbers and the sites of origin of the droplets expelled during expiratory activities. Edinb Med J, 52(11), 385-401

2. Johnson, G. R., \& Morawska, L. (2009). The mechanism of breath aerosol formation. J Aerosol Med Pulm Drug Deliv, 22(3), 229-237. http://dx.doi.org/10.1089/jamp.2008.0720

3. Scheuch, G. (2020). Breathing is enough: for the spread of influenza virus and SARS-CoV-2 by breathing only. Journal of aerosol medicine and pulmonary drug delivery, 33(4), 230-234

4. Bourouiba, L. (2020). Turbulent Gas Clouds and Respiratory Pathogen Emissions: Potential Implications for Reducing Transmission of COVID-19. JAMA, 323(18), 1837-1838.

http://dx.doi.org/10.1001/jama.2020.4756

5. Comber, L., E, O. M., Drummond, L., Carty, P. G., Walsh, K. A., De Gascun, C. F., et al. (2020). Airborne transmission of SARS-CoV-2 via aerosols. Rev Med Virol, e2184. http://dx.doi.org/10.1002/rmv.2184

6. Dudalski, N., Mohamed, A., Mubareka, S., Bi, R., Zhang, C., \& Savory, E. (2020). Experimental investigation of far-field human cough airflows from healthy and influenza-infected subjects. Indoor Air. http://dx.doi.org/10.1111/ina.12680

7. Helgeson, S. A., Lim, K. G., Lee, A. S., Niven, A. S., \& Patel, N. M. (2020(ja)). Aerosol Generation during Spirometry. Ann Am Thorac Soc. http://dx.doi.org/10.1513/AnnalsATS.202005-569RL. 
8. Mittal, R., Ni, R., \& Seo, J-H. (2020). The flow physics of COVID-19.Journal of fluid Mechanics. ;894.

9. Nissen, K., Krambrich, J., Akaberi, D., Hoffman, T., Ling, J., Lundkvist, A., et al. (2020). Long-distance airborne dispersal of SARS-CoV-2 in COVID-19 wards. Sci Rep, 10(1), 19589.

http://dx.doi.org/10.1038/s41598-020-76442-2

10. Santarpia, J. L., Rivera, D. N., Herrera, V. L., Morwitzer, M. J., Creager, H. M., Santarpia, G. W., et al. (2020). Aerosol and surface contamination of SARS-CoV-2 observed in quarantine and isolation care. Scientific reports, 10(1), 1-8

11. Van Doremalen, N., Bushmaker, T., Morris, D. H., Holbrook, M. G., Gamble, A., Williamson, B. N., et al. (2020). Aerosol and surface stability of SARS-CoV-2 as compared with SARS-CoV-1. New England Journal of Medicine, 382(16), 1564-1567

12. Wilson, N. M., Norton, A., Young, F. P., \& Collins, D. W. (2020). Airborne transmission of severe acute respiratory syndrome coronavirus-2 to healthcare workers: a narrative review. Anaesthesia, 75(8), 10861095. http://dx.doi.org/10.1111/anae.15093

13. Nieß, A., Bloch, W., Friedmann-Bette, B., Grim, C., Gärtner, B., Halle, M., et al. (2020). Recommendations for Exercise Testing in Sports Medicine during the Current Pandemic Situation (SARS-CoV-2/COVID-19).

14. ARTP. Covid19 infection control issues for lung function 2020 [Available from: https://www.artp.org.uk/News/covid19-infection-control-issues-for-lung-function.

15. Crimi, C., Impellizzeri, P., Campisi, R., Nolasco, S., Spanevello, A., \& Crimi, N. (2020). Practical considerations for spirometry during the COVID-19 outbreak: Literature review and insights. Pulmonology. http://dx.doi.org/10.1016/j.pulmoe.2020.07.011

16. Faghy, M. A., Sylvester, K. P., Cooper, B. G., \& Hull, J. H. (2020). Cardiopulmonary exercise testing in the COVID-19 endemic phase. Br J Anaesth. http://dx.doi.org/10.1016/j.bja.2020.06.006

17. McGowan, A., Sylvester, K., Burgos, F., Boros, P., de Jongh, F., Kendrick, A., et al.. Recommendation from ERS Group 9.1 (Respiratory function technologists /Scientists) Lung function testing during COVID-19 pandemic and beyond: ERS 9.1; 2020 [Available from: https://ers.app.box.com/s/zs1uu88wy51monr0ewd990itoz4tsn2h.

18. Prachand, V. N., Milner, R., Angelos, P., Posner, M. C., Fung, J. J., Agrawal, N., et al. (2020). Medically Necessary, Time-Sensitive Procedures: Scoring System to Ethically and Efficiently Manage Resource Scarcity and Provider Risk During the COVID-19 Pandemic. J Am Coll Surg, 231(2), 281-288. http://dx.doi.org/10.1016/j.jamcollsurg.2020.04.011

19. Rasam, S. A., Apte, K. K., \& Salvi, S. S. (2015). Infection control in the pulmonary function test laboratory. Lung India, 32(4), 359-366. http://dx.doi.org/10.4103/0970-2113.159571

20. World Health Organization. Report of the WHO-China Joint Mission on Coronavirus Disease 2019 (COVID19) 2020 [Available from: https://www.who.int/docs/default-source/coronaviruse/who-china-jointmission-on-covid-19-final-report.pdf.

21. World Health Organization. WMA Declaration of Helsinki - Ethical Princiiples for Medical Research Involving Human Subjects 2013 [Available from: https://www.wma.net/policies-post/wma-declaration-ofhelsinki-ethical-principles-for-medical-research-involving-human-subjects/.

22. Vyaire Medical. AirLife Misty Fast disposable small-volume nebulizer (SVN), Competitive Analysis. 
23. Ching, I., \& Dunn-Rankin, D. (1996). Light scattering measurements of cigarette smoke. Aerosol Sci Tech, 24, 85-101

24. Bake, B., Larsson, P., Ljungkvist, G., Ljungstrom, E., \& Olin, A. C. (2019). Exhaled particles and small airways. Respir Res, 20(1), 8. http://dx.doi.org/10.1186/s12931-019-0970-9

25. Fairchild, C. I., \& Stampfer, J. F. (1987). Particle concentration in exhaled breath. Am Ind Hyg Assoc J, 48(11), 948-949. http://dx.doi.org/10.1080/15298668791385868

26. Morawska, L., Johnson, G., Ristovski, Z., Hargreaves, M., Mengersen, K., Corbett, S., et al. (2009). Size distribution and sites of origin of droplets expelled from the human respiratory tract during expiratory activities. Journal of Aerosol Science, 40(3), 256-269

27. O'Neil, C. A., Li, J., Leavey, A., Wang, Y., Hink, M., Wallace, M., et al. (2017). Characterization of Aerosols Generated During Patient Care Activities. Clin Infect Dis, 65(8), 1335-1341.

http://dx.doi.org/10.1093/cid/cix535

28. Papineni, R. S., \& Rosenthal, F. S. (1997). The size distribution of droplets in the exhaled breath of healthy human subjects. J Aerosol Med, 10(2), 105-116. http://dx.doi.org/10.1089/jam.1997.10.105

29. Fabian, P., Brain, J., Houseman, E. A., Gern, J., \& Milton, D. K. (2011). Origin of exhaled breath particles from healthy and human rhinovirus-infected subjects. J Aerosol Med Pulm Drug Deliv, 24(3), 137-147. http://dx.doi.org/10.1089/jamp.2010.0815

30. Savory, E., Lin, W. E., Blackman, K., Roberto, M. C., Cuthbertson, L. R., Scott, J. A., et al. (2014). Western Cold and Flu (WeCoF) aerosol study-preliminary results. BMC Res Notes, 7(1), 563.

http://dx.doi.org/10.1186/1756-0500-7-563

31. Tang, J. W., Nicolle, A. D., Klettner, C. A., Pantelic, J., Wang, L., Suhaimi, A. B., et al. (2013). Airflow dynamics of human jets: sneezing and breathing-potential sources of infectious aerosols. Plos One, 8(4), e59970

32. Asadi, S., Wexler, A. S., Cappa, C. D., Barreda, S., Bouvier, N. M., \& Ristenpart, W. D. (2019). Aerosol emission and superemission during human speech increase with voice loudness. Sci Rep, 9(1), 2348. http://dx.doi.org/10.1038/s41598-019-38808-z

33. Edwards, D. A., Man, J. C., Brand, P., Katstra, J. P., Sommerer, K., Stone, H. A., et al. (2004). Inhaling to mitigate exhaled bioaerosols. Proc Natl Acad Sci U S A, 101(50), 17383-17388.

http://dx.doi.org/10.1073/pnas.0408159101

\section{Tables}

Table 1. Volunteer Test Subject Demographic Data

\begin{tabular}{llllll} 
Subject & Sex & Age (years) & Race & Height $(\mathbf{c m})$ & Weight $(\mathbf{k g})$ \\
\hline PP1 & Male & 56 & Caucasian & 170 & 102 \\
\hline PP2 & Male & 45 & Caucasian & 173 & 82
\end{tabular}

Table 2. Breath-by-Breath CPET Subject Exercise Data during Resting Breathing and Peak Exercise 


\begin{tabular}{lllllllll} 
Subject & $\begin{array}{l}\text { CPET } \\
\text { Interface }\end{array}$ & $\begin{array}{l}\text { CPET } \\
\text { Sensor }\end{array}$ & $\begin{array}{l}\text { Resting } \\
\text { Breathing } \\
\text { Minute } \\
\text { Ventilation } \\
(\mathrm{L} / \mathrm{min})\end{array}$ & $\begin{array}{l}\text { Peak } \\
\text { Exercise } \\
\text { Minute } \\
\text { Ventilation } \\
(\mathrm{L} / \mathrm{min})\end{array}$ & $\begin{array}{l}\text { Resting } \\
\text { Breathing } \\
\text { Tidal } \\
\text { Volume } \\
(\mathrm{L})\end{array}$ & $\begin{array}{l}\text { Peak } \\
\text { Exercise } \\
\text { Tidal } \\
\text { Volume } \\
(\mathrm{L})\end{array}$ & $\begin{array}{l}\text { Peak } \\
\text { Exercise } \\
\text { Load } \\
(\text { W) }\end{array}$ & $\begin{array}{l}\text { Peak } \\
\text { Exercise } \\
\text { HR } \\
(\mathrm{BPM})\end{array}$ \\
\hline PP1 & Headgear & MFS & 29.0 & 143.7 & 1.4 & 2.7 & 255 & 137 \\
\hline PP2 & Headgear & MFS & 28.3 & 149.4 & 1.6 & 3.5 & 279 & 179 \\
\hline PP1 & Mask & DVT & 31.8 & 125.4 & 1.4 & 2.4 & 261 & 141 \\
\hline PP2 & Mask & DVT & 23.8 & 145.9 & 1.7 & 3.1 & 282 & 181
\end{tabular}

CPET: cardiopulmonary exercise testing; W: watts; HR; heart rate; BPM: beats per minute; MFS: mass flow sensor; DVT: digital volume transducer.

\section{Figures}



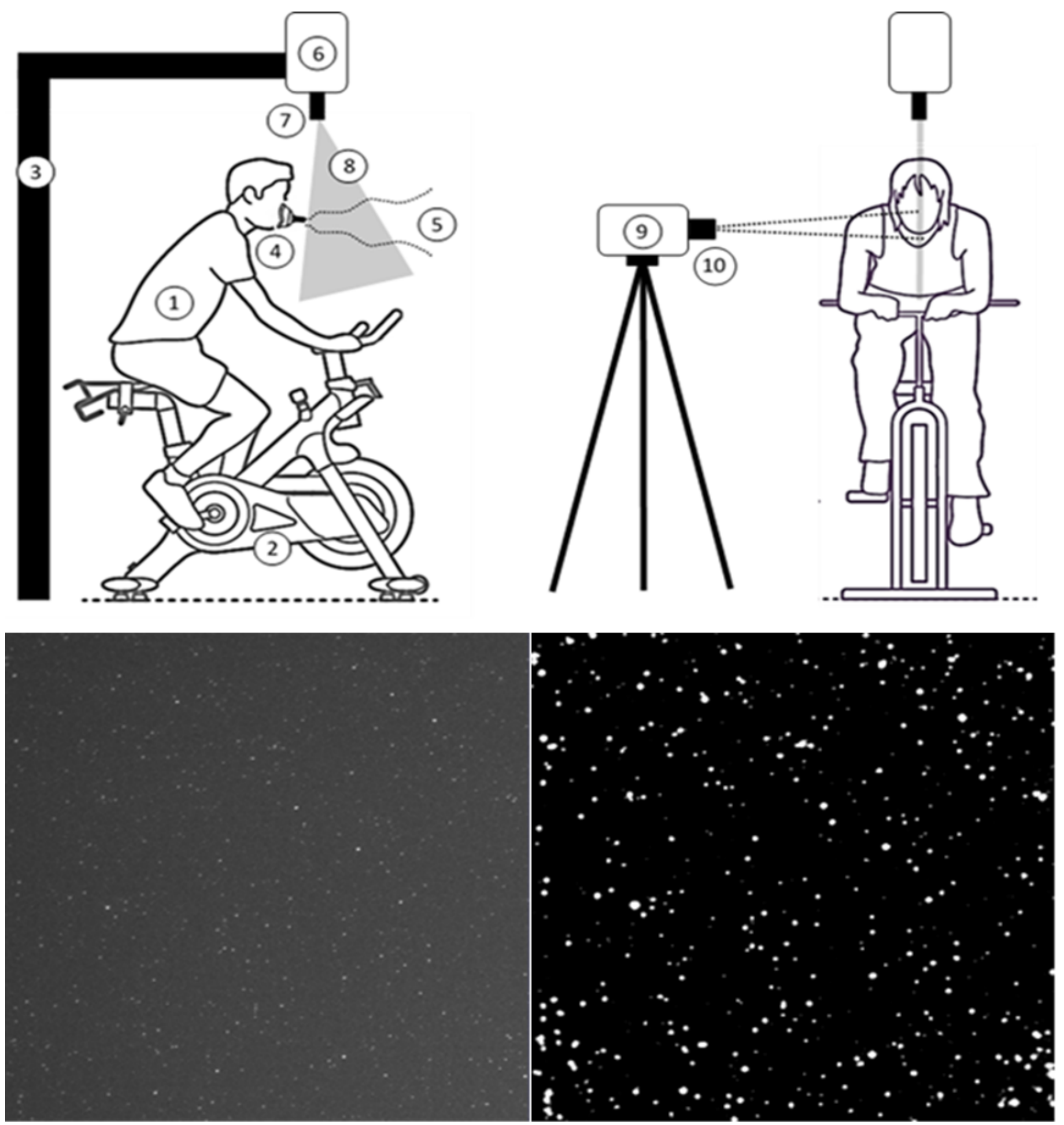

Figure 1

Top - Experimental Test Setup: 1) Subject 2) Exercise Bike 3) Gantry 4) CPET Interface 5) Breath Region 6) Pulsed-Laser 7) Sheet Optics 8) Laser Sheet 9) CMOS Camera 10) Camera Lens. Bottom - Image Processing Example - Raw Image (left), Processed Image (right). Multi-step smoothing and pixel threshold algorithm consisted of: a) subtracting the statistical minimum pixel intensity from each image to remove the background; b) smoothing each image to eliminate pixel noise; and c) identification and counting of particles based upon pixel intensity threshold. 


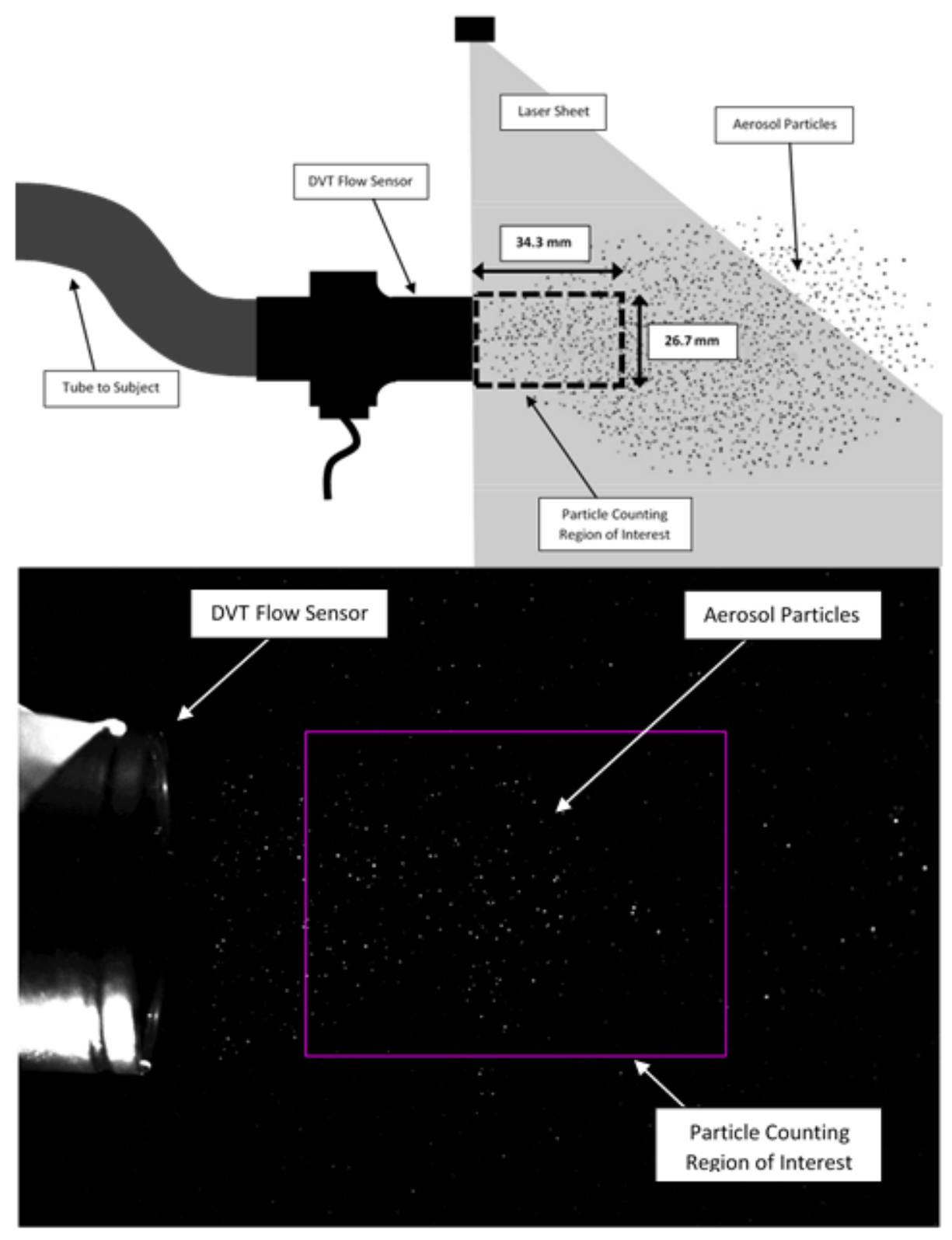

\section{Figure 2}

CPET Breath-by-Breath Experimental Measurement Setup (Top) and Image of Aerosol Particle Counting Region (Bottom) with Digital Volume Transducer 


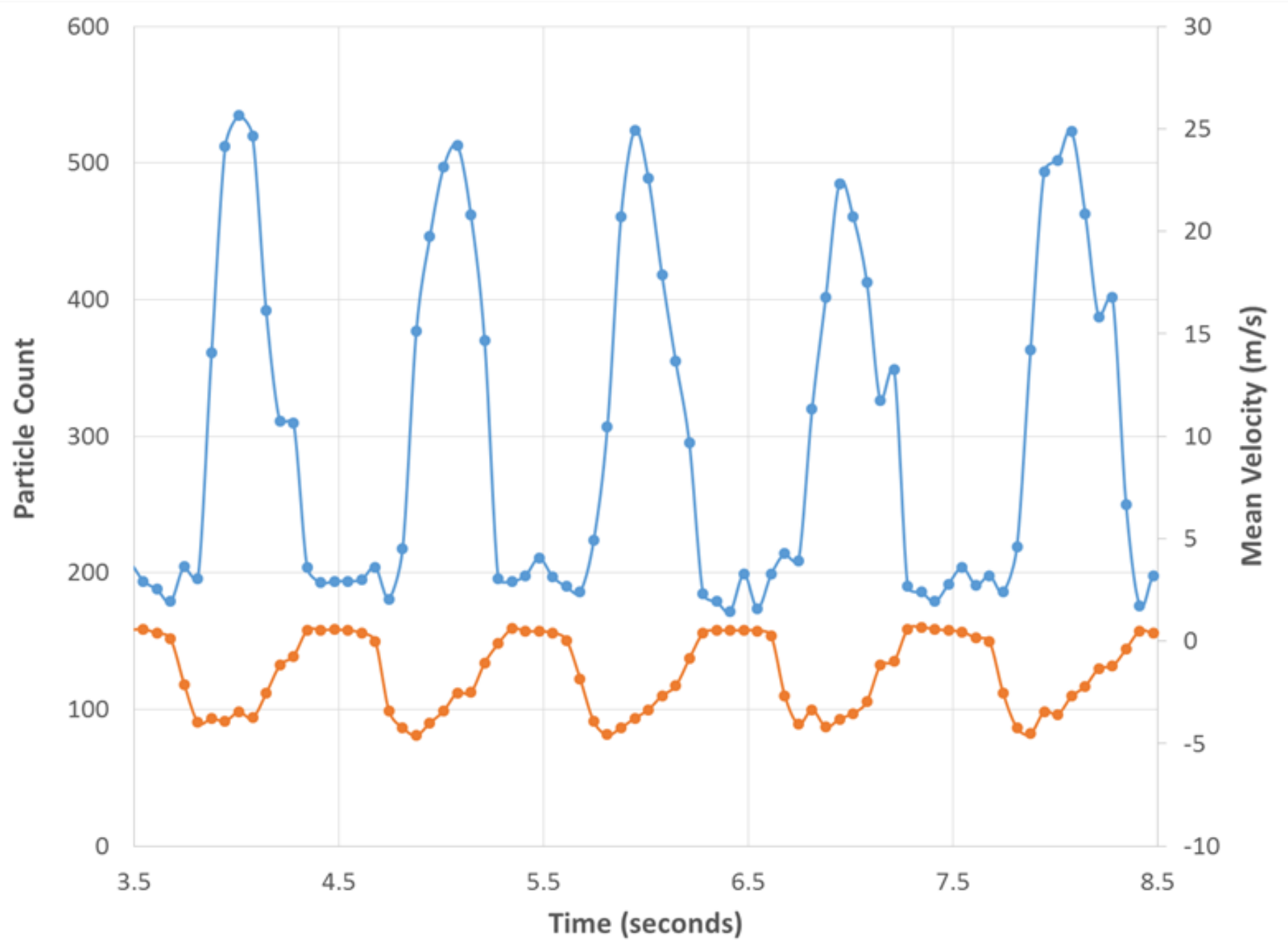

Figure 3

CPET Breath-by-Breath Raw Particle Count (Blue) and Mean Velocity (Orange) as a function of Time at Peak Exercise Intensity, Subject PP1. Particle counts rise to a maximum during exhalation and return to a baseline value during inhalation. Transition from inhalation to exhalation occurs as mean velocity transitions from positive to negative values.
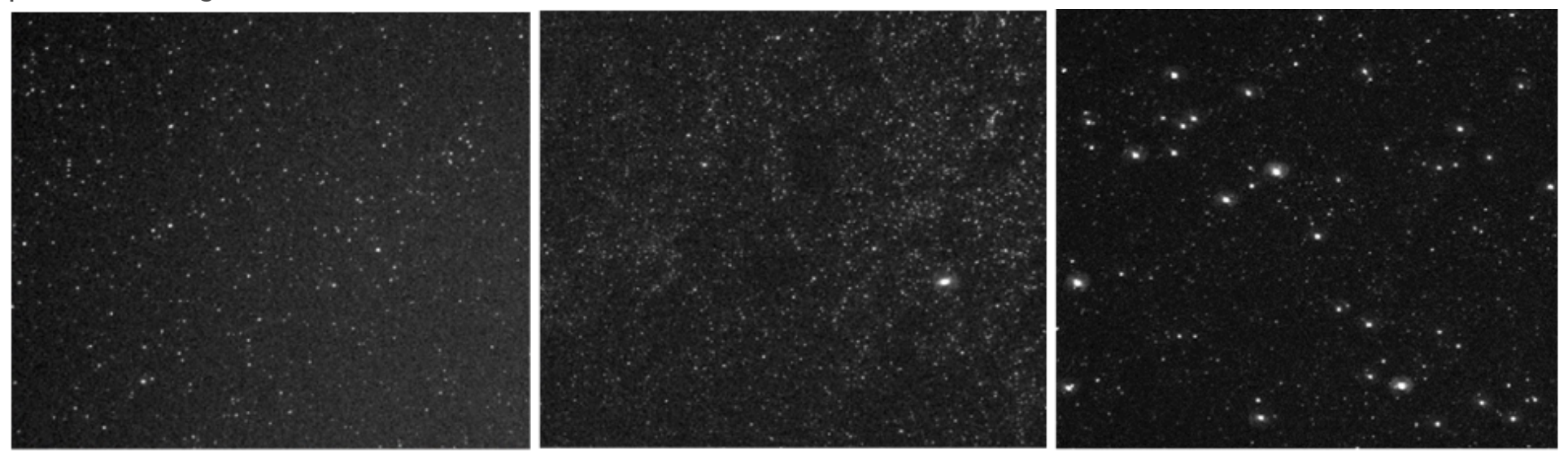

Figure 4 
Comparison of CPET Aerosol Particles (left) with Tobacco Smoke Particles (center) and Jet Nebulizer Aerosol Droplets (right)

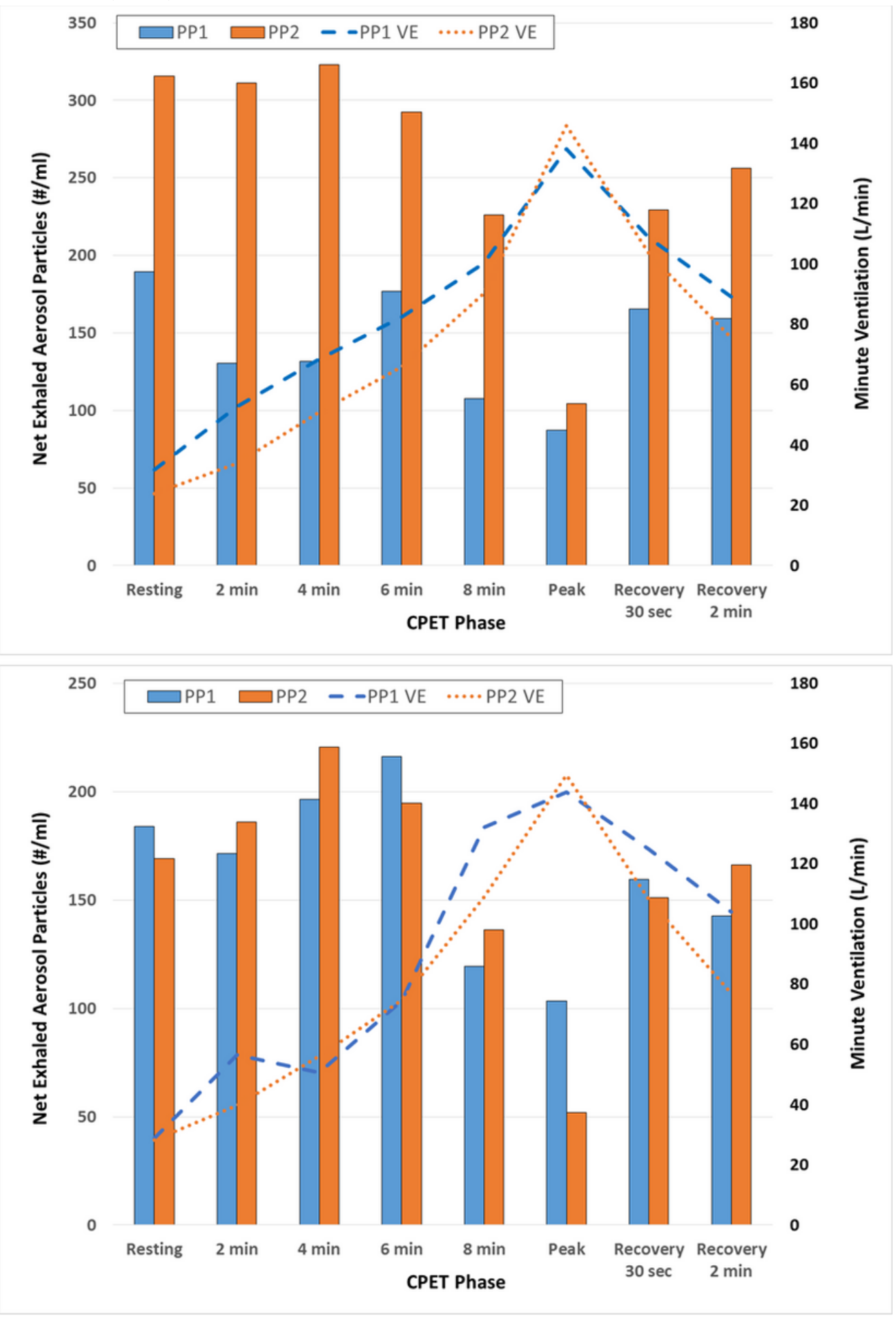

Figure 5

NEP per ml and Minute Ventilation (VE) as a Function of CPET Phase (DVT - top, MFS - bottom) NEP: net exhaled particles; CPET: cardiopulmonary exercise testing; VE: minute ventilation; MFS: mass flow sensor; DVT: digital volume transducer. 


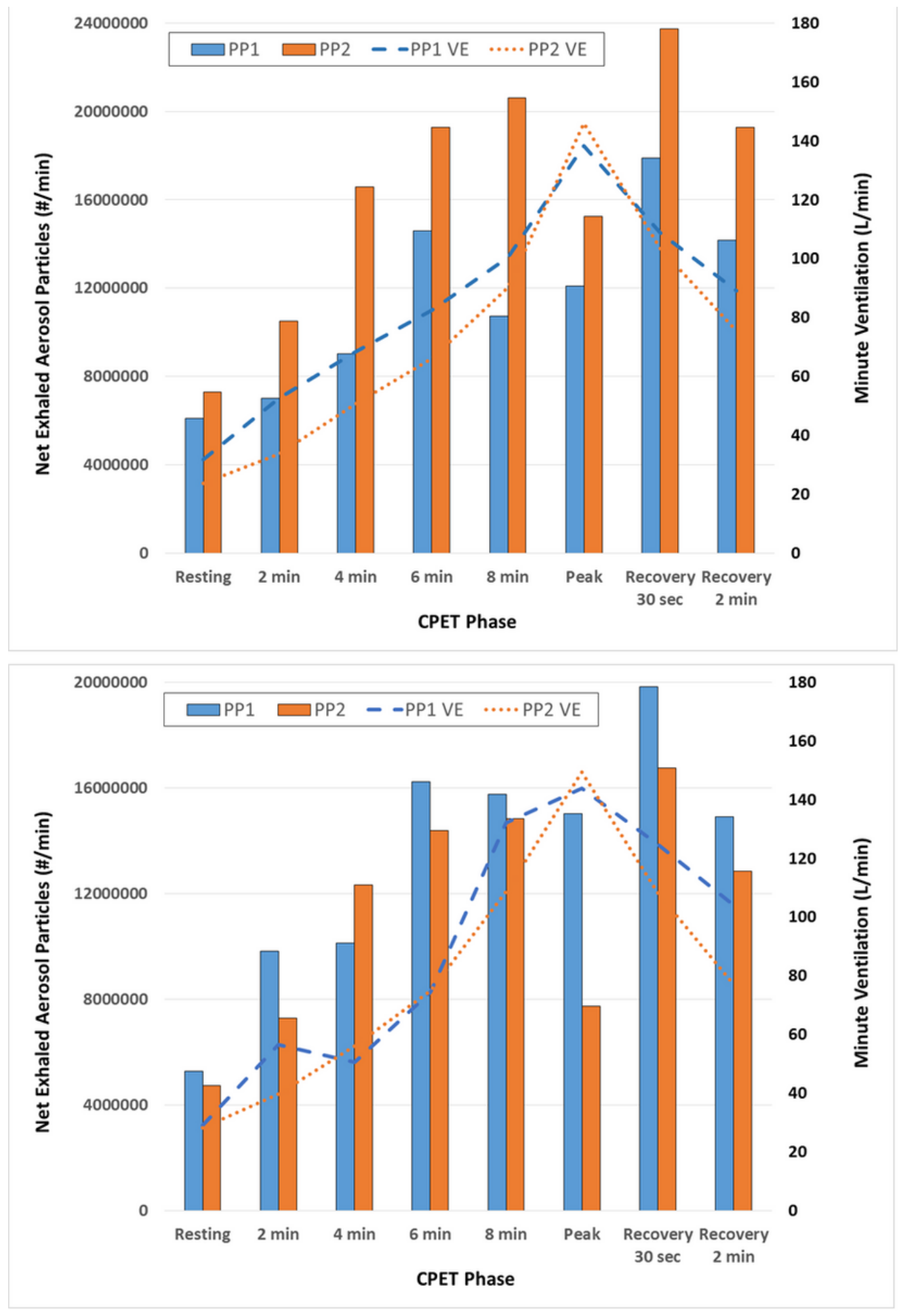

Figure 6

NEP per Minute and Minute Ventilation as a Function of CPET Phase (DVT - top, MFS - bottom) NEP: net exhaled particles; CPET: cardiopulmonary exercise testing; VE: minute ventilation; MFS: mass flow sensor; DVT: digital volume transducer. 


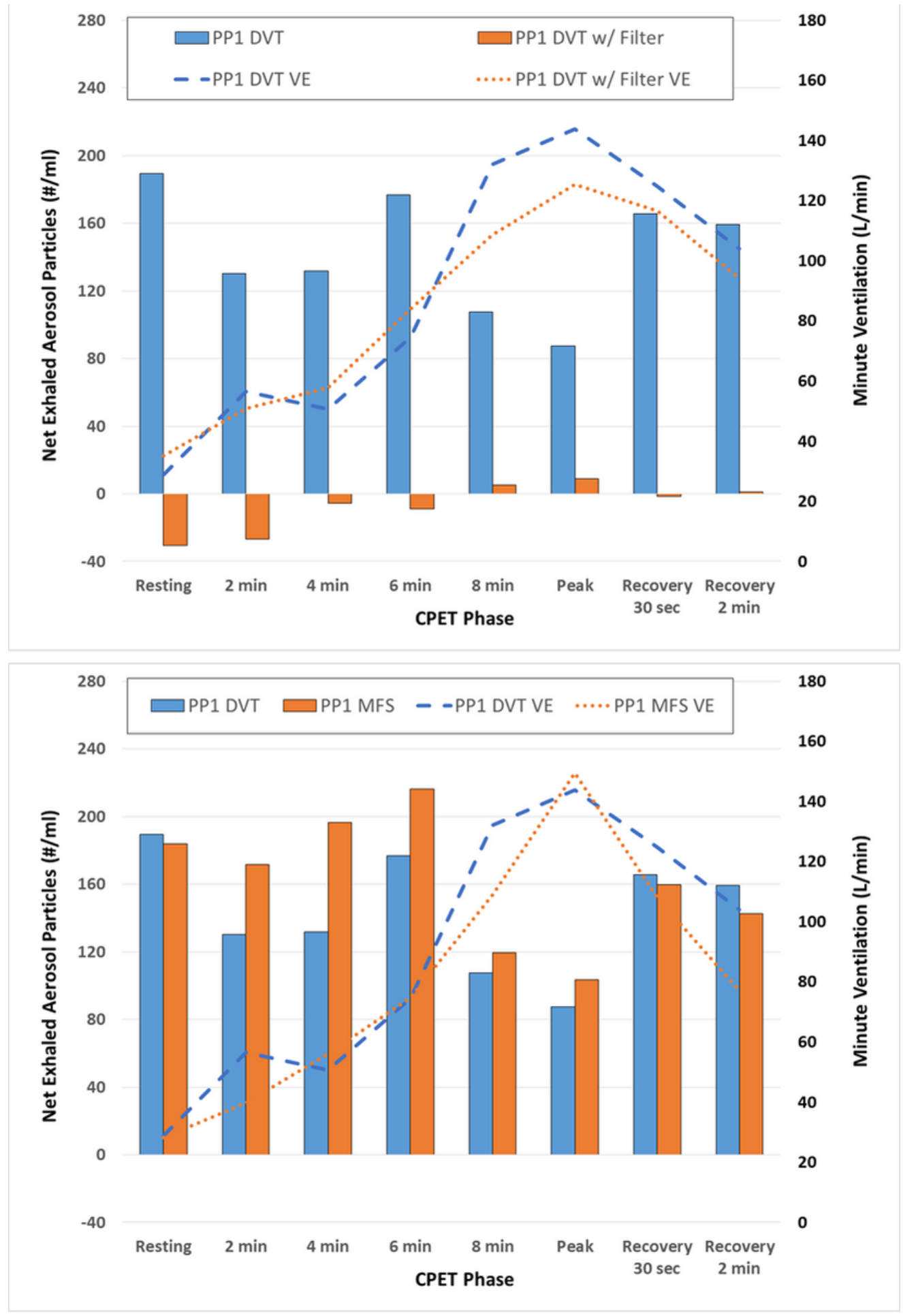

Figure 7

NEP per $\mathrm{ml}$ as a Function of CPET Phase - PP1, DVT versus MFS NEP: net exhaled particles; CPET: cardiopulmonary exercise testing; VE: minute ventilation; MFS: mass flow sensor; DVT: digital volume transducer. 